\title{
Comparison of Various Sitting Postures on Pulmonary Function, Lumbar Curvature, and Comfort Evaluations
}

\author{
Chun-Ting Li, Chih-Han Chang, Jheng-Hung Huang, and Kuen-Horng Tsai
}

\begin{abstract}
This study aims to explore the effects on pulmonary function, lumbar curve, and comfort evaluations in the postures such as slumped sitting, lumbar support sitting, sacrum support sitting, and slanting sitting.

10 healthy people were recruited to join this study. An experimental chair was innovated so that the regions corresponding to thoracic vertebrae, lumbar vertebrae, and sacrum were adjustable separately. The researchers then employed gas analyzers, goniometer, and comfort evaluation questionnaires to collect data and conduct statistic analysis.

The results show that pulmonary function and comfort evaluation in sacrum support sitting posture appear to be substantially better than all the others. Regarding lumbar angle, lumbar support sitting posture results in a significant lordosis, and is closer to the lumbar curve in standing posture.
\end{abstract}

Index Terms-Posture, lumbar, wheelchairs, rehabilitation.

\section{INTRODUCTION}

Poor sitting position has been a common problem for wheelchair users [1], which effects of the pulmonary function and spine curvature. Many recent studies have suggested that physical postures and pulmonary functions are associated [2]-[14]. The study of Chen et al. examined the function of pulmonary relating to angles between trunk and thighs of patients with spinal injuries sitting on wheelchairs. The results showed that pulmonary would be functioning better at an angle, between the trunk and thighs, larger than 90 degree. Another study by Lalloo et al. explored the function of pulmonary in standing and sitting postures of healthy people. The results suggested that pulmonary would be functioning better in the standing posture than that in the sitting one [15]. Lin et al. found that respiratory function was subject to the sitting posture [16]. In the study, the researchers measured forced vital capacity (FVC), forced expiratory volume in 1 second (FEV1), and peak expiratory flow (PEF) in three postures. The results revealed that the respiratory function was better in the standing posture, which would improve respiratory function. Therefore, remaining lordotic helps improving the respiratory function.

A wheelchair seating system is important for wheelchair users. It affects their postural support, muscle activity, pressure relief, pulmonary function, mobility, and comfort.

Manscript received March 15, 2014; revised May 15, 2014.

Chun-Ting Li and Chih-Han Chang are with the Institute of Biomedical Engineering, National Cheng Kung University, No.1, University Road, Tainan City 701, Taiwan (e-mail: p8897109@mail.ncku.edu.tw, cchang@mail.bme.ncku.edu.tw) .

Jheng-Hung Huangn and Kuen-Horng Tsai are with Graduate Institute of Mechatronic System Engineering, National University of Tainan, No. 33, Sec. 2, Shu-Lin St., Tainan 70005, Taiwan (e-mail: ctli@ymail.com, tsaikh@mail.nutn.edu.tw).
The standard sling seat and back upholstery were frequently used for the wheelchair users, but the function of maintaining optimal postural position was limited.

Given that, it is plausible to state that a good sitting posture may improve pulmonary function. The purpose of this research is to explore whether sitting postures produce any influences on the pulmonary function, the lumbar curve, and the comfort evaluations. Four sitting postures to be examined are slumped sitting, lumbar support sitting, sacrum support sitting, and slanting sitting. The research team hoped to find out the best sitting posture that can be singled out to help wheelchair users.

\section{Methods}

\section{A. Subjects}

10 healthy people were chosen to take part in this experiment, with an average age of $24.7 \pm 1.49 \mathrm{yr}$, an average height of $172.6 \pm 8.68 \mathrm{~cm}$, an average weight of $72.1 \pm 11.92 \mathrm{~kg}$, and an average BMI value of $24.21 \pm 3.58$. Their ages, genders, heights, and weights were categorized prior to the beginning of the experiment to ensure the data collected meet the requirements. Every subject had to complete a personal information form and sign a written consent form with the following conditions: no history of any known spinal disease, or low back pain; normal range of motion (ROM) with spine, no discomfort of pain with spinal motion; and no severe perceptual or cognitive impairment that may affect the experiment. The experiment has been approved by the panel of human subject research of National University of Tainan.

\section{B. Experimental Chair}

The back of the chair in this experiment was divided into three regions, which were the thoracic vertebrae region, the lumbar vertebrae region, and the sacrum region. A supporting mechanism was installed in each region with a back-and-forth stroke of $6.5 \mathrm{~cm}$, an up-and-down stroke of 3 $\mathrm{cm}$, and a rotation of 30 degrees. Each mechanism can be manually adjusted by a separate wire control knob as shown in Fig. 1 and Fig. 2.

\section{Postures (Fig. 3)}

A slumped sitting posture is the one which the upper part of the user leans fully on the back of chair, with lumbar vertebrae pushing greatly backwards, pelvis pressing firmly against back supporter, spine curving to bulge out. To achieve a lumbar support sitting posture, on the experimental chair, one must let the adjusting mechanism push forward 6 $\mathrm{cm}$ on the subject's L3 vertebra with the subject's back resting against the back supporter. In this experiment, a sacrum support sitting posture defined as the one which the sacrum was pushed $6 \mathrm{~cm}$ forward to the subject's sacrum 
region on the experimental chair. The lumbar support mechanism was adjusted $5 \mathrm{~cm}$ forward and its surface at an angle fully pressed on the lumbar curve. The subject's thoracic vertebrae was in a naturally comfortable state. A slanting sitting posture is the one which the back of the chair is lowered until the angle between seat and back is at the 135 degrees. The subject got seated with his/her back resting on the back support. As for a standing posture, the subject had to keep his/her body in a natural shape, in which cervical vertebrae and lumbar vertebrae were posed forward with thoracic vertebrae, and sacrum backward.

In all sitting postures, knee joints were supposed to be at the 90 degrees and perpendicular to the ground. A space between knee pits and seat had to be left, and the soles of feet must be able to stamp flat on the ground.

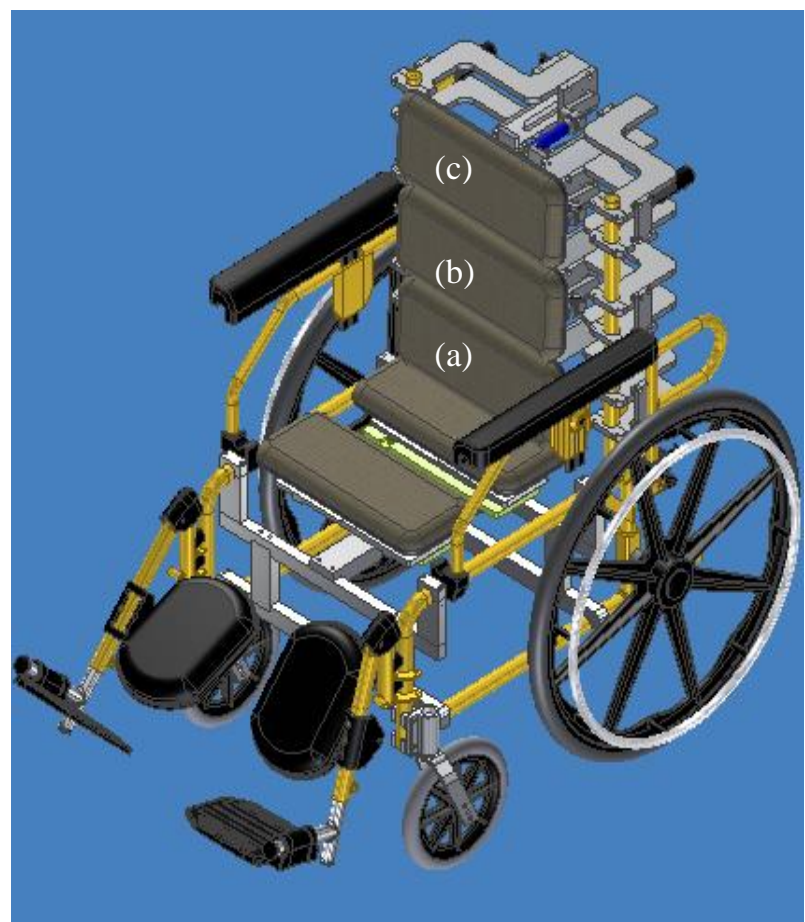

Fig. 1. The experimental wheelchair, (a) the adjustable sacral support, (b) the adjustable lumbar support and (c) the adjustable thoracic support.

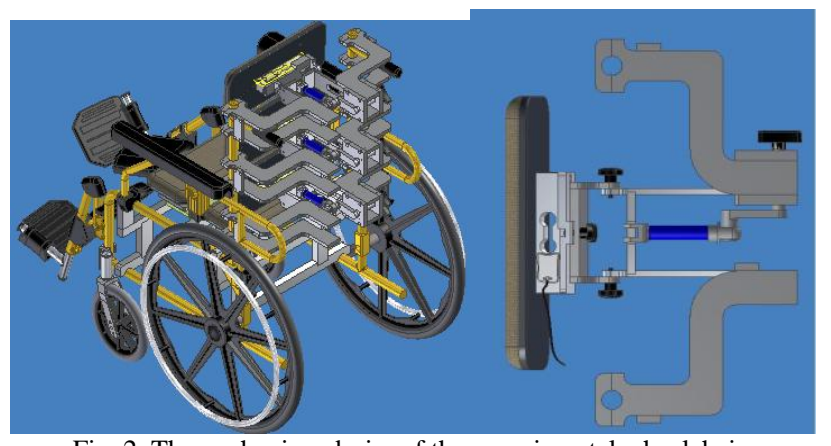

Fig. 2. Themechanism designof the experimental wheelchair.

\section{Procedure}

The gas analysis system called MetaMax ${ }^{\circledR}$ 3B, CORTEX, Germany, was employed to evaluate the pulmonary function. It was used to measure forced vital capacity (FVC), forced expiratory volume in 1 second (FEV1), peak expiratory flow (PEF), and forced expiratory flow rate (FEF25\% 75\%). Through a facemask and a sampling tube, this device collected the exhaled gas of the subject. The gas sensor analyzes the respiratory function. During the experiment, 5 normal cycles of breath were a prologue to a formal process in which the subject must inhale deeply to fill pulmonarys with the air, and then exhale slowly until the zero gas flow. The exhalation process should exceed 6 seconds, which is the standard duration for the pulmonary function measurement.

The goniometer SG150/B and SG150 made by Biometrics Ltd, UK, was employed to measure lumbar vertebras curve with supports at different regions. The angles between lumbar vertebrae and sacrum in the sacrum support sitting posture were measured and analyzed, and later were compared with the data obtained from the other three sitting postures. The purpose was to discover whether the spinal angle of the subject in sacrum support sitting posture would be close to the spinal angle in the standing posture. The electronic principle of the device is that its resistance values vary with trunk angles. The analogue data can be turned into a digital display by accessing the software on InstruNet.

With regard to the comfort evaluation, a questionnaire was sent to the ten subjects to inquire about the differences in various sitting postures. It started with the evaluation of spinal curve, in which the first section began with the evaluation of the overall comfort (including muscles) on spine in the four sitting postures. The second section dealt with the evaluation of the comfort on thoracic vertebrae in the four sitting postures. The third section was on the lumbar vertebrae in the same circumstances. The fourth section was on the sacrum-lumbar vertebrae regions in the same circumstances. The fifth section which explored how smooth was the subject's breathing in the four sitting postures was about the evaluation of breathing. The final section involved the evaluation of the subject's view. There were five options, ranging from $\mathrm{A}$ to $\mathrm{E}$ in every question where $\mathrm{A}$ is the highest score of 5 .

\section{E. Statistical Analysis}

We opted for the SPSS 17.0 as our analytical application to carry out the statistical analysis of various evaluations. In addition, analysis of variance (ANOVA) was also employed on the assumption that each group of variance was equal. Therefore, Levene's test had to be used to assess the divergence of the sub-regions in different postures. In the process of Post-hoc test, Tukey's test was employed in conjunction on the pairwise assessment to check whether there were divergences in the mean values between any pair. The $\mathrm{P}$ value was 0.05 .

\section{RESULT}

Table I shows the measurement results of pulmonary function. The FVC, FEV1, PEF, FEF25\%, FEF50\%, FEF75\%, and FEF25\%-75\% in sacrum support sitting posture are better than the other sitting postures $(P<0.05)$, in terms of respiratory efficiency, and is close to the respiratory efficiency in the standing posture $(P<0.05)$.

Table II is the measurement results of spine angles, which shows that when lumbar shift, respiratory efficiency alters correspondingly. The correlation between lumbar positions has been illustrated in the table. In sacrum support sitting posture with the sacrum supporter stuck out and lumbar vertebrae supported, the pressure on the subject's diaphragm might also be altered. As it turns out the vital capacity is 
expanded correspondingly, the symptoms of hypoventilation, secretion residue in bronchiole, and atelectasis can also be

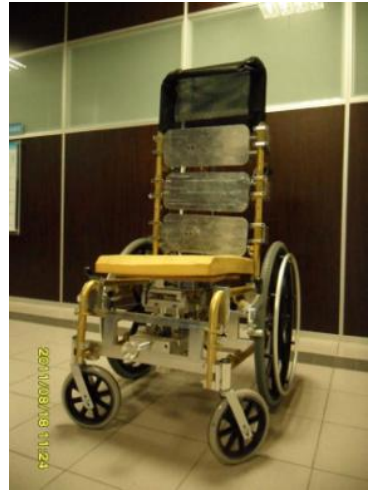

(A)

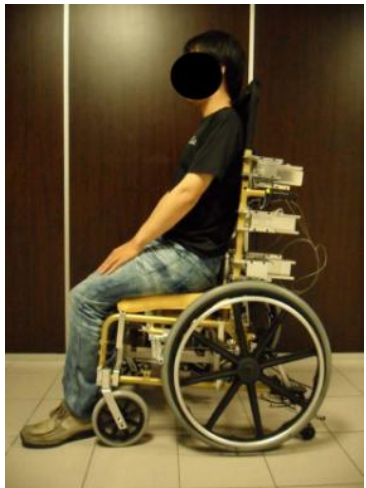

(D)

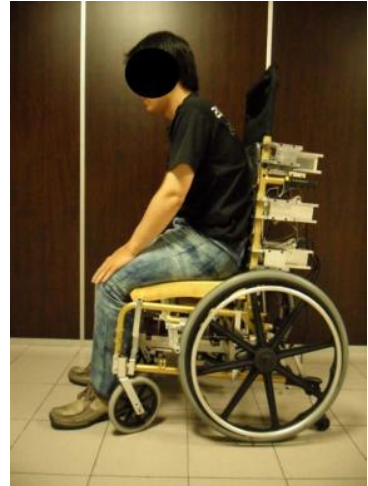

(B)

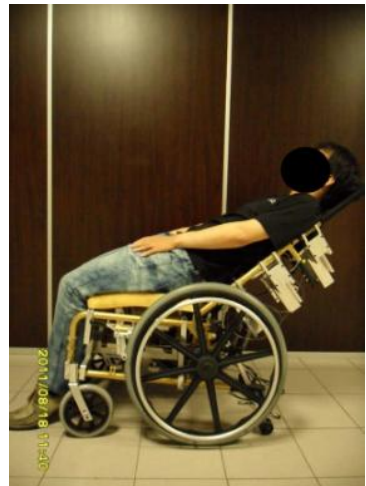

(E)

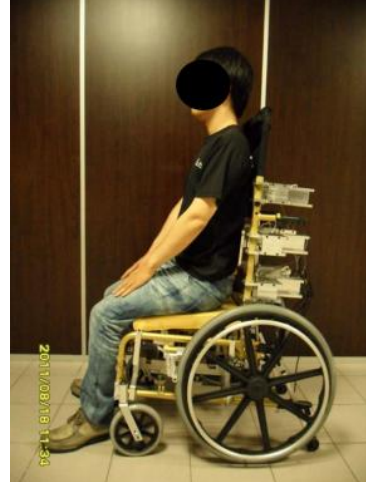

(C)

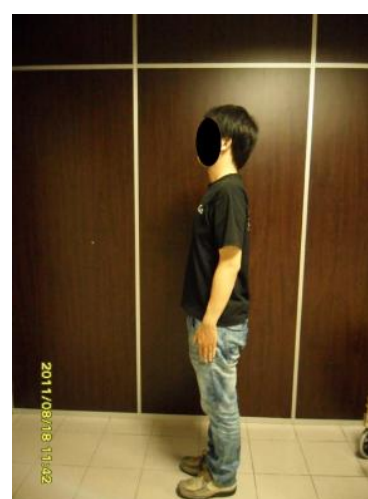

(F)

Fig. 3. (A) experimental chair, (B) slumped sitting posture, (C) lumbar support sitting posture, (D) sacrum support sitting posture, (E) slanting sitting posture, and $(\mathrm{F})$ standing posture

TABLE I: LUNG FUnCTIONS IN DIFFERENT SITTING POSTURES

\begin{tabular}{lccccc}
\hline & Slumped & Lumbar support & Sacrum support & Slanting & Standing \\
\hline FVC (1) & $1.75 \pm 0.8$ & $2.03 \pm 0.54$ & $2.64 \pm 0.86$ & $2.04 \pm 1.21$ & $2.73 \pm 0.8$ \\
FEV1(1) & $1.07 \pm 0.46$ & $1.16 \pm 0.35$ & $1.53 \pm 0.57$ & $1.24 \pm 0.49$ & $1.49 \pm 0.49$ \\
PEF (1/s) & $1.54 \pm 0.54$ & $1.66 \pm 0.52$ & $2.01 \pm 0.7$ & $1.68 \pm 0.57$ & $2.12 \pm 0.68$ \\
FEF25 75\% (1/s) & $1.29 \pm 0.48$ & $1.35 \pm 0.44$ & $1.73 \pm 0.66$ & $1.37 \pm 0.54$ & $1.74 \pm 0.48$ \\
FEF75\% (1/s) & $1.47 \pm 0.49$ & $1.45 \pm 0.45$ & $1.85 \pm 0.69$ & $1.54 \pm 0.55$ & $1.94 \pm 0.61$ \\
FEF50\% (1/s) & $1.44 \pm 0.53$ & $1.35 \pm 0.44$ & $1.72 \pm 0.59$ & $1.44 \pm 0.58$ & $1.84 \pm 0.57$ \\
FEF25\% (1/s) & $1.08 \pm 0.45$ & $1.06 \pm 0.39$ & $1.29 \pm 0.41$ & $1.11 \pm 0.49$ & $1.28 \pm 0.36$ \\
\hline & & TABLE III: RESULTS OF LUMBAR ANGLE MEASUREMENT & & Slanting \\
\hline
\end{tabular}

Table III is the results of comfort evaluations. Considering the overall comfort, the sacrum support sitting posture provided the best comfort $(P<0.05)$. The thoracic region was adequately supported in the sacrum support sitting posture; therefore, the highest comfort was provided in the sacrum support sitting posture. Regarding the lumbar vertebrae comfort, the lumbar support sitting posture was exceedingly upright with no supporter in the regions of thoracic vertebrae and sacrum-pelvis. The ten subjects were experienced discomfort in the lumbar vertebrae region when sitting for a long time. While in the sacrum support sitting posture, the lumbar regions were adequately supported; therefore, it could guarantee superior comfort in the lumbar region. As a result, the sacrum support sitting posture is of highest comfort. In addition, in every sitting posture the sacrum-pelvis region is always supported, and lumbar gets sufficient rest because the lumbar region is mildly slanting. Therefore, the comfort evaluation on this item in all sitting postures got higher scores, among which comfort of sacrum gets the highest score in the sacrum support sitting posture. Regarding respiratory smoothness, the scores in the sacrum support and the lumbar support postures were close to each other because lumbar got substantial support in the lumbar vertebrae support sitting posture and the spinal curve was close to that in the standing 
posture. In addition, the lumbar regions were adequately supported in the sacrum support sitting posture, which contributes to better respiratory smoothness. For visual acuity, it was the same in both the sacrum support and the lumbar vertebrae support sitting posture. Both of the sitting postures could keep the spinal curve upright and provide better visual acuity. This research explored the effects of different four sitting postures on pulmonary, lumbar curve, and comfort evaluation on comfort. The results suggest that further clinical researches would help wheelchair users.

TABLE III: RESULTS OF COMFORT SCORES IN DIFFERENT SITTING POSTURES

\begin{tabular}{|c|c|c|c|c|}
\hline & Slumped & Lumbar support & Sacrum support & Slanting \\
\hline Overall comfort & $2.4 \pm 0.97$ & $3.5 \pm 0.53$ & $4.4 \pm 0.52$ & $3.9 \pm 0.88$ \\
\hline comfort in thoracic vertebrae & $2.4 \pm 0.70$ & $2.7 \pm 0.82$ & $4.2 \pm 0.63$ & $3.6 \pm 1.07$ \\
\hline Comfort in lumbar vertebrae & $2.8 \pm 1.14$ & $3.1 \pm 0.74$ & $4.2 \pm 0.63$ & $3.4 \pm 0.84$ \\
\hline Comfort in sacrum vertebrae & $3.2 \pm 0.79$ & $3.4 \pm 0.84$ & $4.2 \pm 0.79$ & $3.7 \pm 0.95$ \\
\hline Respiratory smoothness & $2.2 \pm 0.63$ & $4.1 \pm 0.57$ & $4.2 \pm 0.42$ & $3.7 \pm 1.06$ \\
\hline Visual acuity & $2.7 \pm 1.34$ & $4.1 \pm 0.88$ & $4.1 \pm 0.88$ & $3.3 \pm 1.16$ \\
\hline
\end{tabular}

\section{DISCUSSION}

Recently, because of the progress of medical technology and the maturity of health care, the life-span of the wheelchair user is almost the same as people in general. However, most of the users are likely to experience low back pain, pressure sore and the respiratory dysfunction. Until now, there is no suggestion from medical views on sitting postures that can resolve all the above problems. The wheelchair-users still suffer from the pain. This study points out how a sitting posture could improve the above difficulties and benefit for the wheelchair-users.

Based on the analysis on the pulmonary function, lumbar curve, and the comfort evaluation, it can be concluded that the sacrum support sitting posture stood out as the best one to provide better respiratory efficiency in comparison with all the other three sitting postures, which are slanting sitting, slump sitting, and upright sitting. The results suggest that a sitting posture could improve respiratory efficiency, which, in turn, may also offer a better sitting posture for patients with impaired lower limbs.

\section{CONCLUSION}

It can be concluded that there is a relationship between a sitting posture and the pulmonary function, lumbar curvature, and comfort of a wheelchair user. This study found a new sitting posture can generate a better performance on pulmonary function and mentality. Furthermore, the new sitting posture can have the same effect of standing posture. As to the spinal angle, lumbar upright sitting posture is the most similar to standing posture because of better spinal curve.

In order to make sure the new sitting posture can improve the health conditions, this study compared it with the lumbar upright sitting posture, the slumped sitting posture, the tilt sitting posture, and the standing posture. This study used a new type of pressure relax wheelchair which can be adjusted based on thoracic, lumbar and sacral. Thepulmonary function, the spinal angle and the mentality of the subject were analyzed quantitatively and qualitatively to find out the differences of each posture.

\section{REFERENCES}

[1] D. Zacharkow, "Posture: Sitting, standing, chair design and exercise," Springfield (IL), Charles C Thomas, pp. 237-309, 1988.

[2] C. Chen, I. Lien, and M. Wu, "Respiratory function in patients withspinal cord injuries: Effects of posture," Paraplegia, vol. 28, pp. 81-86, 1990

[3] D. A. Hobson and R. E. Tooms, "Seated lumbar/pelvic alignment. A comparison between spinal cord-injured and noninjured groups," Spine, vol. 17 , pp. $293-8,1992$.

[4] U. G. Lalloo, M. R. Becklake, and C. M. Goldsmith, "Effect of standing versus sitting position on spirometric indices in healthy subjects," Respiration, vol. 58, pp. 122-5, 1991.

[5] W. J. Crosbi and S. Myles, "An investigation into effect of posturalmodification on some aspect of normal pulmonary function," Physiotherapy, vol. 71, pp. 311-314,1985.

[6] A. Baydur, R. Adkins, and J. Milic-Emili, "Lung mechanics in individualswith spinal cord injury: Effects of injury level and posture," J Appl. Physiol., vol. 90, pp. 405-411, 2001.

[7] M. Appel, A. Childs, E. Healey, S. Markowitz, S. Wong, and J. Mead. "Effect of posture on vital capacity," J. Appl. Physiol., vol. 61, pp. 1882-1884, 1986.

[8] M. Landers, G. Barker, S. Wallentine, J. W. McWhorter, and C. Peel, "Acomparison of tidal volume, breathing frequency, and minuteventilation between two sitting postures in healthy adults," Physiother. Theory Pract., vol. 19, pp. 109-119,2003.

[9] M. Makhsous, J. Bankard, F. Lin, S. Taylor, J. Pedersen, J. Hilb, and R. Hendrix, "Lung capacity and airflow change due to different sitting posture," presented at $27^{\text {th }}$ International Conference on Rehabilitation Engineering and Assistive Technology, Society of North America, Orlando, FL, 2004.

[10] F. Manning, E. Dean, J. Ross, "Effects of side lying onlung function in older individuals," Phys. Therap., vol. 79, pp. 456-466, 1999.

[11] O. M. Nwaobi and P. D. Smith, "Effect of adaptive seating on pulmonaryfunction of children with cerebral palsy," Dev. Med. Child Neurol., vol. 28, pp. 351-354, 1986.

[12] M. Townsend, "Spirometric forced expiratory volumes measuredin the standing versus the sitting posture," Am. Rev. Respir. Dis., vol. 130, pp. 123-124, 1984.

[13] G. Vilke, T. Chan, T. Neuman, and J. Clausen, "Spirometry in normalsubjects in sitting, prone, and supine positions," Respir. Care, vol. 45, pp. 407-410, 2000.

[14] A. B. Jackson and T. E. Groomes, "Incidence of respiratory complications following spinal cord injury," Arch Phys Med Rehabil, vol. 75, p. 270, 1994.

[15] U. G. Lalloo, M. R. Becklake, and C. M. Goldsmith, "Effect of standing versus sitting position on spirometric indices in healthy subjects," Respiration, vol. 58, pp. 122-125, 1991.

[16] F. Lin, S. Parthasarathy, S. Taylor, D. Pucci, R. Hendrix, and M. Makhsous, "Effect of different sitting postures on lung capacity, expiratory flow, and lumbar lordosis," Arch. Phys. Med. Rehabil., vol. 87, pp. 504-509, 2006. 


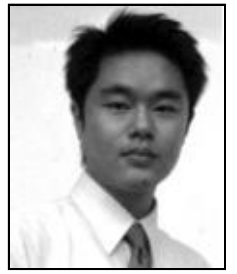

Chun-Ting Li received his MSc from Graduate Institute of Mechatronic System Engineering, National University of Tainan, Taiwan, in 2006 and 2008. He is currently a $\mathrm{PhD}$ candidate of Institute of Biomedical Engineering, National Cheng Kung University, Taiwan. His major studies involve biomechanics, rehabilitation engineering, human factors, and biomedical engineering.

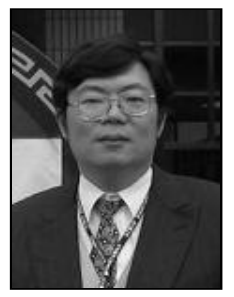

Chih-Han Chang received his $\mathrm{MSc}$ and $\mathrm{PhD}$ degrees from the Department of Mechanical Engineering, Rice University, USA, in 1986 and 1990, respectively. He is currently a professor at Institute of Biomedical Engineering, National Cheng Kung University, Taiwan. His major studies involve biomedical engineering, biomechanics, and rehabilitation engineering. Dr. Chang is a member of the Biomedical Engineering Society.

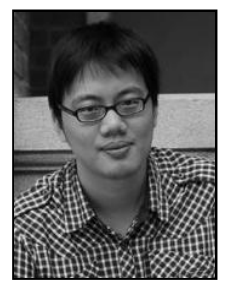

Jheng-Hung Huang received his MSc from Graduate Institute of Mechatronic System Engineering, National University of Tainan, Taiwan, in 2008 and 2010. He is currently a mechatronic system engineer of Innolux Corporation, Taiwan. His major studies involve biomechanics, rehabilitation engineering, human factors, biomedical engineering, and mechatronic system engineering.

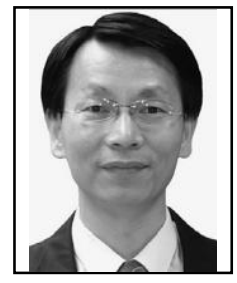

Kuen-Horng Tsai received his MSc and $\mathrm{PhD}$ degrees from Institute of Biomedical Engineering, National Cheng Kung University, Taiwan, in 1991 and 1997, respectively. He is currently the chief of Innovation Incubation Center and a professor at Institute of System Engineering, National University of Tainan, Taiwan. His major studies involve rehabilitation engineering and biomedical engineering, biomechanics. 